\title{
Periodic driving control of Raman-induced spin-orbit coupling in Bose-Einstein condensates: the heating mechanisms
}

\author{
J.M. Gomez Llorente and J. Plata \\ Departamento de Física, Universidad de La Laguna, \\ La Laguna E38204, Tenerife, Spain.
}

\begin{abstract}
We focus on a technique recently implemented for controlling the magnitude of synthetic spinorbit coupling (SOC) in ultra-cold atoms in the Raman-coupling scenario. This technique uses a periodic modulation of the Raman-coupling amplitude to tune the SOC. Specifically, it has been shown that the effect of a high-frequency sinusoidal modulation of the Raman-laser intensity can be incorporated into the undriven Hamiltonian via effective parameters, whose adiabatic variation can then be used to steer the SOC. Here, we characterize the heating mechanisms that can be relevant to this method. We identify the main mechanism responsible for the heating observed in the experiments as basically rooted in driving-induced transfer of population to excited states. Characteristics of that process determined by the harmonic trapping, the decay of the excited states, and the technique used for preparing the system are discussed. Additional heating, rooted in departures from adiabaticity in the variation of the effective parameters, is also described. Our analytical study provides some clues that may be useful in the design of strategies for curbing the effects of heating on the efficiency of the control methods.
\end{abstract}

PACS numbers: 67.85.-d, 32.10.Fn, 33.60.+q, 37.10.Gh 


\section{INTRODUCTION}

The realization of spin-orbit coupling (SOC) in Bose-Einstein condensates has opened the way to intense experimental and theoretical work which has brought about significant advances in the research on ultra-cold atoms [1-3]|. Variations of the original setup, including schemes applicable to fermionic systems [4, 5], have been developed, and, a series of fundamental effects have been realized. Envisaged technical implications of this extended scenario, in particular, potential applicability to create novel states of matter, like topological insulators or nontrivial superfluids, are being explored [1, 2]. Apart from enriching the dynamics in diverse forms, SOC can be considered as an element to manipulate it. Especially relevant to the strategies of control, is the recent realization of a technique to steer in real time the characteristics of SOC. It was proposed that, in the Raman-laser coupling setup [1], the application of a high-frequency sinusoidal modulation of the laser intensity can be used to convert the system into an effective undriven counterpart where the adiabatic variation of the modulation amplitude can serve to control the strength of the SOC [6]. The experimental realization confirmed the applicability of this scheme: the detected response of the system agreed with the theoretical predictions on the changes in the SOC strength. The system was also observed to present heating dependent on the driving frequency [7]. Here, we extend the previously applied theoretical framework to account for the heating mechanisms. Apart from the practical interest of improving the approach that supports the control method, our study has fundamental implications: it deals with the development of models for a complex scenario with elements of general relevance. Different stages, associated with increasing precision in the modeling, can be singled out in our description. We will proceed by isolating first the essential components of the heating processes, and, dealing, subsequently, with specific aspects of the considered setup. A primary difficulty is rooted in the required treatment of the atomic interactions. Indeed, as the experimental preparation corresponded to a condensate, departures from the single-particle dynamics must be evaluated. Further complexity is introduced by the synthetic coupling of internal and external degrees of freedom. Although there have been significant advances in the analysis of the

effect of the SOC on the dynamics of a condensate [8-10], the studies have frequently concentrated on a uniform scenario and on features associated with the lowest band and with low-energy excitations. Here, the need of dealing with the inter-band transitions induced 
by the (high-frequency) driving implies focusing on additional elements. The evaluation of the rate of population transfer to excited states requires explicitly taking into account the harmonic trapping. Moreover, a detailed description of the dispersion relations of the elementary excitations in different energy ranges is needed. We must also deal with damping of the excited states, and, therefore, with the role of nonlinear inter-mode coupling. The restriction to a particular set of SOC parameters will allow us to cope with the system complexity, and, still, identify basic mechanisms responsible for the observed features. From the obtained insight, some useful clues to setting up methods for curbing the effects of heating may be extracted. Beyond the control objectives, our description opens the way to the design of strategies for exploring different aspects of the SOC systems.

The outline of the paper is as follows. In Sec. II, central elements of our methodology are introduced: through an appropriate unitary transformation, we set up a perturbative scheme to deal with the sinusoidal driving of the Raman coupling amplitude. The perturbative parameter will be shown to be given by the quotient between the modulation amplitude of the Raman-coupling strength and the modulation frequency. In Sec. III, a single-particle approach is presented to simulate general characteristics of the experimental findings. The role of perturbative corrections to the scenario of control proposed in Ref. [7] is assessed. In particular, we focus on the first-order term, which accounts for driving-induced transfer of population to excited states. Atomic-interaction effects are tackled in Sec. IV: we deal with ground-state properties, elementary excitations, and damping. Special attention is given to the dependence of the heating process on decay of the excitations resulting from inter-mode coupling. In Sec. V, features specific to the ramp implemented to prepare the system are analyzed. Finally, some general conclusions are summarized in Sec. VI.

\section{CONTROL OF THE SPIN-ORBIT COUPLING}

We consider the realization of SOC in Bose-Einstein condensates reported in Ref. [1]. There, an appropriate arrangement of Raman lasers was used to create synthetic SOC, specifically, to couple one of the components of the external linear momentum of the atoms p to an effective "spin", i.e., to a two-level internal system formed by hyperfine states. The setup was composed by two orthogonally polarized Raman lasers with different frequencies and propagation directions. The arrangement characteristics were designed to couple two 
Zeeman-split internal states, and, in particular, to make the coupling dependent on the atom external momentum. In this form, an effective SOC was induced. In a single-particle approach, and, ignoring the effect of the harmonic confinement, that scenario is described by the Hamiltonian

$$
H=\left(\frac{\hbar^{2} k_{x}^{2}}{2 m}+E_{L}\right) I+\frac{\hbar \Omega_{0}}{2} \sigma_{x}+\left(\frac{\hbar \delta_{0}}{2}+\alpha_{0} k_{x}\right) \sigma_{z}
$$

where $m$ is the atomic mass, $I$ is the unit matrix, $\sigma_{i}(i=x, y, z)$ are the Pauli matrices corresponding to the pseudospin, i.e., to the considered effective two-level system, and $k_{x}$ denotes the quasi-momentum in the coupling direction $(\mathbf{p}=\hbar \mathbf{k})$. Additionally, concerning the laser characteristics, $\Omega_{0}$ denotes the Raman coupling amplitude, $\delta_{0}$ represents an adjustable detuning, and $\alpha_{0}$ is the strength of the realized SOC $\left(\alpha_{0}=2 E_{L} / k_{L}\right.$ where $E_{L}=\hbar^{2} k_{L}^{2} / 2 m$ is the recoil energy and $k_{L}=2 \pi \sin (\theta / 2) / \lambda$ is the recoil momentum; $m$ is the atomic mass, $\lambda$ is the laser wavelength, and, $\theta$ is the angle between the Raman lasers, which, here, as in the experiments, is taken to be $\theta=\pi / 2[1,11])$.

Let us see how the SOC strength can be controlled by modulating the Raman coupling amplitude in the form

$$
\Omega_{0} \rightarrow \Omega(t)=\Omega_{0}+\Omega_{R} \cos (\omega t)
$$

Appropriate to analyze the dynamics resulting from the driving is the application of the unitary transformation

$$
U(t)=\exp \left[-\frac{i}{2} \frac{\Omega_{R}}{\omega} \sin (\omega t) \sigma_{x}\right] .
$$

The transformed Hamiltonian, given by

$$
H^{\prime}=U^{\dagger} H U-i \hbar U^{\dagger} \dot{U}
$$

is written, after straightforward algebra [12], as

$$
\begin{aligned}
H^{\prime}= & \left(\frac{\hbar^{2} k_{x}^{2}}{2 m}+E_{L}\right) I+\frac{\hbar \Omega_{0}}{2} \sigma_{x}+ \\
& \left(\frac{\hbar \delta_{0}}{2}+\alpha_{0} k_{x}\right)\left\{\cos [\zeta(t)] \sigma_{z}+\sin [\zeta(t)] \sigma_{y}\right\},
\end{aligned}
$$


where

$$
\zeta(t) \equiv \frac{\Omega_{R}}{\omega} \sin (\omega t)
$$

Now, taking into account the expansion of $\cos [\zeta(t)]$ and $\sin [\zeta(t)]$ in terms of the ordinary Bessel functions $J_{n}(u)$ [13], we can rewrite the Hamiltonian $H^{\prime}$ as the sum of different-order contributions. This approach provides us with a perturbative scheme where different-order corrections to the scenario of control proposed in Ref. [7] can be evaluated. Note that the small parameter in the perturbative approach is determined by $\Omega_{R} / \omega$. The zero-order term, given by

$$
H_{0}=\left(\frac{\hbar^{2} k_{x}^{2}}{2 m}+E_{L}\right) I+\frac{\hbar \Omega_{0}}{2} \sigma_{x}+J_{0}\left(\frac{\Omega_{R}}{\omega}\right)\left(\frac{\hbar \delta_{0}}{2}+\alpha_{0} k_{x}\right) \sigma_{z},
$$

has the same functional form as the undriven Hamiltonian $H$. The parameters $\delta_{0}$ and $\alpha_{0}$, present in $H$, have been replaced here by their effective counterparts $\delta=J_{0}\left(\frac{\Omega_{R}}{\omega}\right) \delta_{0}$ and $\alpha=J_{0}\left(\frac{\Omega_{R}}{\omega}\right) \alpha_{0}$. The offset $\Omega_{0}$ is the same in both Hamiltonians. The energy bands corresponding to $H_{0}$ are given by

$$
E_{ \pm}\left(k_{x}\right)=\frac{\hbar^{2} k_{x}^{2}}{2 m}+E_{L} \pm \sqrt{\left(\frac{\hbar \Omega_{0}}{2}\right)^{2}+J_{0}^{2}\left(\frac{\Omega_{R}}{\omega}\right)\left(\frac{\hbar \delta_{0}}{2}+\alpha_{0} k_{x}\right)^{2}}
$$

$H_{0}$ provides the framework used in the design of the technique of control: the modification in real time of the effective SOC strength $(\alpha)$ and detuning $(\delta)$ can be achieved by adiabatically varying the modulation amplitude $\Omega_{R}$.

The validity of this scheme was tested in Ref. [7]. There, the dynamics of a condensate, prepared in the lowest band, was monitored at different values of $\Omega_{R}$. In each case, the measurements were carried out after implementing a linear ramp to adiabatically vary $\Omega_{R}$ from zero to its final value. We stress that the efficiency of the control method depends strongly on the character of the system evolution. An adiabatic following of the lowest eigenstate of the changing Hamiltonian is needed for the method to be efficient.

Different aspects of the comparison between the theoretical predictions and the experimental results must be stressed. First, the values of SOC strength, determined by directly measuring the momentum distribution, were shown to agree with those predicted by the model. Second, the system was also found to present heating, parametrized in terms of the temperature in the presentation of the results. This effect was particularly relevant in 
a central range of driving frequencies frequencies, namely, for $2 \mathrm{kHz}<\omega / 2 \pi<7 \mathrm{kHz}$. No explanation of these heating effects was given by the theory. However, the authors presented a qualitative analysis, using a Floquet-state formalism, which provided some clues on the found stability of the system at large and small frequencies, and on the instability at intermediate frequencies. Apart from the energy bands as function of the driving frequency, the spatial density distributions were obtained. See the Supplemental Material in Ref. [7]. The explanation of the heating effects, and, in general, the departure of the system dynamics from the picture given by $H_{0}$, is tackled in the next section.

\section{SINGLE-PARTICLE DESCRIPTION OF THE HEATING MECHANISMS}

Here, we will concentrate on general features of the heating that can be traced to the single-particle dynamics. Although, we will basically deal with the uniform scenario described in the previous section, we will incidentally refer to differential aspects of the role of the harmonic confinement. Many-body interaction effects will be incorporated into the description further on.

\section{A. Inter-band transfer of population induced by the driving}

Crucial to the description of the heating detected in the experiments is the inclusion in our approach of the first-order correction to $H_{0}$, given by

$$
H_{1}=2 J_{1}\left(\frac{\Omega_{R}}{\omega}\right) \sin (\omega t)\left(\frac{\hbar \delta_{0}}{2}+\alpha_{0} k_{x}\right) \sigma_{y} .
$$

It is apparent that, provided that the driving frequency is quasi-resonant with the interband splitting, i.e., for $\hbar \omega \sim E_{+}\left(k_{x}\right)-E_{-}\left(k_{x}\right), H_{1}$ can represent an efficient mechanism of population transfer from the lowest energy-band $E_{-}\left(k_{x}\right)$ to the excited band $E_{+}\left(k_{x}\right)$. It is important to take into account that, since $H_{1}$ commutes with $p_{x}$, the transition conserves the momentum: it corresponds to a vertical line in a diagram of energy bands versus the quasimomentum $k_{x}$. When the harmonic confinement is considered, the coupling induced by $H_{1}$ becomes more complex since the eigenstates do not have a well-defined momentum. Given the weakly-harmonic confinement realized in practice, a quasi-continuum approximation is feasible, and the use of the term "band" is still appropriate. The following discussion applies 
also to that case.

Outside the resonance region, no significant transfer of population between the bands is induced by $H_{1}$. In particular, for high driving-frequencies, $\hbar \omega \gg E_{+}\left(k_{x}\right)-E_{-}\left(k_{x}\right)$, the stability of the system in the lowest band is guaranteed, and, consequently, the proposed strategy of control is sound. Indeed, $H_{0}$ provides then a satisfactory (coarse-grained) description of the dynamics: the higher-order (oscillating) terms in the expansion of $H^{\prime}$, which are given by

$$
H_{n}=2 J_{n}\left(\frac{\Omega_{R}}{\omega}\right) \cos (n \omega t)\left(\frac{\hbar \delta_{0}}{2}+\alpha_{0} k_{x}\right) \sigma_{i}^{(n)},(n \geq 1)
$$

where $\sigma_{i}^{(n)}=\sigma_{z}$ when $n$ is even, and $\sigma_{i}^{(n)}=\sigma_{y}$ when $n$ is odd, can be averaged out to zero since the driving period $2 \pi / \omega$ is much smaller than any other relevant time scale in the dynamics. The applicability of the averaging method is supported by the fact that, as $\Omega_{R} / \omega$ decreases, the functions $J_{n}\left(\Omega_{R} / \omega\right)(n \geq 1)$ diminish and eventually go to zero. In turn, as the magnitude of the perturbative terms decreases, the associated time scale grows, allowing the coarse graining over the driving period. In the opposite regime, namely, for $\hbar \omega \ll E_{+}\left(k_{x}\right)-E_{-}\left(k_{x}\right)$, an adiabatic approximation can be implemented in the original driven Hamiltonian, i.e., in $H$ with $\Omega_{0}$ replaced by $\Omega(t)$. The system, initially prepared in the lowest band, evolves following adiabatically the lowest eigenstate of $H(t)$. Consequently, no inter-band transfer of population takes place either in this range. Note the differences existent between this adiabatic regime, associated with a low driving-frequency $\omega$, and that applied in the control method, characterized by a slow variation of the driving amplitude $\Omega_{R}$, compatible with a high driving-frequency.

From the above description, the observed emergence of instability in the quasi-resonant region is explained as resulting from the transfer of population to the quasi-continuum of states of the upper band. The additional role of the decay of the excited state will be taken into account later on. In Ref. [7], a numerical study based on the Floquet formalism with a reduced number of states simulated the unstable behavior as a crossing between bands. Our characterization of the term that induces the loss of population allows identifying precisely the spectral region where that behavior becomes more relevant. Additionally, our analytical approach paves the way for designing a strategy for stabilizing the system: one can think of fitting $\Omega_{R} / \omega$ to one of the zeros of $J_{1}\left(\Omega_{R} / \omega\right)$ in order to suppress the perturbative inter- 
band coupling. To validate this proposal, we must guarantee that the rest of the terms in the expansion of the Hamiltonian do not significantly contribute to heating. This is the case in the addressed unstable region: it is apparent that, for $\hbar \omega \sim E_{+}\left(k_{x}\right)-E_{-}\left(k_{x}\right)$, those additional terms are out of resonance with the inter-band transition, and, therefore, given their (reduced) magnitude, are irrelevant to population transfer. In contrast, one must contemplate the possibility of having significant heating in a series of spectral windows defined by the conditions $\hbar n \omega \sim E_{+}\left(k_{x}\right)-E_{-}\left(k_{x}\right),(n>1)$, where the different higherorder terms $H_{n}$ are quasi-resonant with the inter-band transition. A detailed analysis of the system response in each case is needed. Further on, we will present results for the magnitude of the different corrections. Still, we can anticipate here a qualitative evaluation of the relative importance of the different terms by addressing a particular situation. Let us consider working conditions where the effect of each of the terms can be expected to be maximum; namely, we assume that, apart from fixing $\omega$ inside the $n$-quasi-resonant region, the parameter $\Omega_{R}$ is adjusted in order to have a maximum of $J_{n}\left(\Omega_{R} / \omega\right)$. From the decreasing magnitude of the maxima as $n$ increases, it is inferred that the relative importance of the different terms in the expansion of the Hamiltonian (at their maximum efficiencies in population transfer), diminishes as the order grows. Here, a general remark on the limitations of the strategy proposed for stabilizing the system is pertinent: although the loss of population can be curbed by reducing $J_{1}^{2}\left(\Omega_{R} / \omega\right)$ via an appropriate choice of the driving parameters, this implies also fixing the SOC parameters in the zero-order Hamiltonian, i.e., loosing the possibility of steering the dynamics.

\section{B. Heating induced by departures from adiabaticity}

In the above analysis, a fixed $\Omega_{R}$, i.e., a concluded adiabatic ramp of $\Omega_{R}$, was assumed. We turn now to deal with a more realistic scenario by explicitly incorporating the ramping process into our approach. In the experiments, a linear ramp was realized; accordingly, here, we consider $\Omega_{R}(t)=\Omega_{R} t / T$, where $T$ denotes the length of the ramp. Moreover, we work in the representation of states with fixed pseudospin component and time-dependent external part, specifically, a plane wave with quasi-momentum following the changing bandminimum. Again, we stress that, although the characterization of the eigenstates becomes more elaborate when harmonic confinement is considered, the following qualitative argu- 
ments are equally valid. The corresponding corrections to the Hamiltonian are obtained by including the specific time dependence of the modulation amplitude in the unitary transformation, i.e., by replacing $\Omega_{R}$ by $\Omega_{R}(t)$ in Eq. (3), and, additionally, by taking into account the variation of the applied basis. The transformed Hamiltonian, obtained from Eq. (4), is given by a more involved expression, which includes the non-adiabatic terms

$$
H_{n a}=-\frac{\hbar \Omega_{R}}{2 T \omega} \sin (\omega t) \sigma_{x}-\frac{\hbar k_{L} \Omega_{R}}{2 T \omega} J_{1}\left(\Omega_{R} t / \omega T\right) x \sigma_{z}, t \leq T
$$

where $x$ is the operator conjugate to $p_{x}$, and, for simplicity, we have taken the particular set of SOC parameters $\delta_{0}=0$ and $\Omega_{0}=0$. The first term comes from the explicit time dependence of $U(t)$ introduced by $\Omega_{R}(t)$. It has a structure similar to that of the previously analyzed driving term, i.e., of that resulting from incorporating the modulation of the Raman amplitude given by Eq. (2) into Eq. (1). Indeed, in the quasi-resonant regime [ $[\hbar \omega \sim$ $\left.E_{+}\left(k_{x}\right)-E_{-}\left(k_{x}\right)\right]$, this term (like $\left.H_{1}\right)$ can lead to heating via interband transfer of population. However, for the considered parameters and ramping rate, the magnitude of the associated effect is $\sim 20$ times smaller than that of the original driving. The second term in Eq. (11) is rooted in the time-dependent character of the motional component of the adiabatic states. Specially relevant is its dependence on $x$, which corresponds to a pseudospin-differentiated dipolar structure. Because of the form of its time dependence, entirely contained in the argument of the Bessel function, this term cannot resonantly couple the bands. However, it can lead to heating via the generation of intra-band excitations. In this sense, it can account for the low-frequency oscillations, observed in the experiments, which were clearly differentiated from the main heating process. In Ref. [7], the origin of those features was already traced to departures from the intended adiabatic following of the ground state induced by imperfections in the realization of the slow ramp. Those oscillations are properly described as collective modes in a many-body approach; their persistence at relatively large times will be analyzed further on. Note that both terms in Eq. (11) consistently vanish in the limit of large ramp-length $T$.

\section{A proposal for evaluating the heating rate applying the Fermi's Golden Rule}

As a first step in the simulation of the experimental results for the heating rate, we propose the analysis of the population transfer from the initial lowest-band state $\left|\varphi^{(i)}\right\rangle$ to the final 
upper-band state $\left|\varphi^{(f)}\right\rangle$ in terms of the Fermi's Golden Rule. Accordingly, after expressing the time-dependent perturbation as $H_{1}=W \sin (\omega t)$, where $W \equiv 2 J_{1}\left(\frac{\Omega_{R}}{\omega}\right)\left(\frac{\hbar \delta_{0}}{2}+\alpha_{0} k_{x}\right) \sigma_{y}$ is a time-independent operator [we consider that the ramp is slow enough to neglect the contribution to interband transfer coming from the first term in Eq. (11)], the transition rate $\Gamma(\omega)$ is written as [14]

$$
\Gamma(\omega)=\frac{\pi}{2 \hbar} \sum_{f}\left|\left\langle\varphi^{(f)}|W| \varphi^{(i)}\right\rangle\right|^{2} \delta\left(E^{(f)}-E^{(i)}-\hbar \omega\right),
$$

with $E^{(i)}$ and $E^{(f)}$ standing, respectively, for the energies of the initial and final states. To evaluate $\Gamma$, we must first identify the states $\left|\varphi^{(f)}\right\rangle$ which, in addition to being connected with $\left|\varphi^{(i)}\right\rangle$ by the perturbation $W$, have energies fulfilling the restriction $E^{(f)}=E^{(i)}+\hbar \omega$. Given the weakly-harmonic confinement realized in practice, a quasi-continuum approximation can be applied to sum the contributions of the set of possible final states. Then, from the form of the density of states and from the dependence of the matrix element $\left\langle\varphi^{(f)}|W| \varphi^{(i)}\right\rangle$ on the different state parameters, the heating rate can be evaluated. Here, the importance of incorporating the harmonic trap into the model must be emphasized. The differences with the uniform scenario are particularly relevant to the evaluation of the required matrix elements. In the uniform case, the transition takes place only at exact resonance between the external frequency and the interband separation. Consequently, $\Gamma(\omega)$ is predicted to be infinitely sharp at this resonance frequency. When the harmonic confinement is considered, the momentum distribution of the ground state widens, and, then, the occurrence of transitions at frequencies different from the resonant one is predicted, (see Fig. 1). Still, a more detailed analysis of the functional form of $\Gamma(\omega)$ is required to explain the results of the experiments, carried out in diverse frequency regimes. For such purpose, additional fundamental aspects of the system, like atom-interaction characteristics, (variations in the form of the ground state, and, damping effects associated with the nonstationary character of the excited states), must be taken into account. Moreover, it is also necessary to tackle the modification of the system parameters that takes place in the ramp implemented to adiabatically vary $\Omega_{R}$ from zero to its final value. The evaluation of the losses in the ramp, where the rate is actually a function of time, is necessary. To incorporate those elements into our framework, we must generalize the above formulation of the Fermi's Golden Rule. This is the objective of the next two sections. 


\section{ATOMIC-INTERACTION EFFECTS IN THE HEATING PROCESSES}

\section{A. Effects associated with ground-state characteristics}

The description of the atomic-interaction effects is required by the experimental conditions, which correspond to the preparation as a condensate. Interaction effects primarily appear in the initial-state wave-function, which becomes dependent on the interaction strength and on the number of particles, and in the excited-mode characteristics, in particular, in the functional form of the dispersion relations. Due to the SOC, the system presents specific components. In particular, there are different interaction strengths associated with the spin combinations. They will be denoted as $g_{\eta, \eta^{\prime}}$, where $\eta$ and $\eta^{\prime}$ refer to the spins. As shown in previous studies, depending on the SOC characteristics and on the values of $g_{\eta, \eta^{\prime}}$, the form of the wave-function of the ground state can display a varied topology [8]. In the case of uniform confinement, three phases, known as stripe, plane-wave (separate dressed-state), and single-minimum phases, can appear. Actually, the description of the system is quite complex in a general regime. Here, we will concentrate on a particular set of SOC parameters, which will allow us to present a simplified picture of the role of interaction effects in heating. Specifically, we will fix the detuning and the offset of the Raman-laser arrangement to the values $\delta_{0}=0$ and $\Omega_{0}=0$. These parameters along with the scattering lengths for the considered system correspond to the regime identified as separate (single-dressed state) phase, which defines the condensation in a single plane-wave state, the sign of the quasimo-

mentum being determined by the particular set of $g_{\eta, \eta^{\prime}}[9,15]$. This restriction, which will be considered also in the analysis of the elementary excitations, is conformable with our aim of identifying mechanisms of general relevance.

The description of the coupling between states, and, in turn, of the whole transfer process can be significantly altered when interaction characteristics are taken into account. It is worth pointing out that the factorization of the initial wave-function and the subsequent reduction in dimensionality applicable to a single-particle system are precluded in the manybody case. Given the form of the coupling term $W$, the distribution of momenta of the initial and final states plays an essential role in the population transfer. Moreover, since some of the experiments were carried out in the high-frequency regime, the characterization of the high-momentum region is particularly important. Note that the analysis of this region is not 
frequent in studies of condensates. These arguments are illustrated in Fig. 1, where results for the heating rate as a function of the driving frequency are presented. For simplicity, an isotropic harmonic trap has been considered. In particular, we have taken the three trap frequencies $\omega_{x}, \omega_{y}$, and $\omega_{z}$, equal to the geometric mean of the frequencies corresponding to the experimental setup [7]. The predictions of the Thomas-Fermi model substantially differ from those of a single-particle approach. Whereas, in the single-particle case, the form of $\Gamma(\omega)$ points to a strong localization of the population transfer in the resonance region, the Thomas-Fermi model accounts for significant heating even at large frequencies. Given the variety of ground-state forms that can emerge as the SOC parameters are changed, it is pertinent to check the robustness of the above findings when the modeling of the ground state is modified. Indeed, as anticipated, we have found that the form of $\Gamma(\omega)$ is strongly dependent on the ground-state characteristics that affect the high momenta. Even so, at this point, we cannot conclusively trace the form of the experimental heating curve to that component of the system: in the following, we will see that the observed widening of $\Gamma(\omega)$ can be rooted also in damping of the excited state. In fact, it will be uncovered that strong damping can make irrelevant the precise modeling of the ground state. To evaluate the relative magnitude of both effects, specifically targeted experiments are required. Note that, in Fig. 1, as in other figures in the article, we have omitted the range of low frequencies where the applied perturbative scheme fails, i.e., when the initially applied unitary transformation and the subsequent averaging procedure do not lead to a useful reduction of the system. 


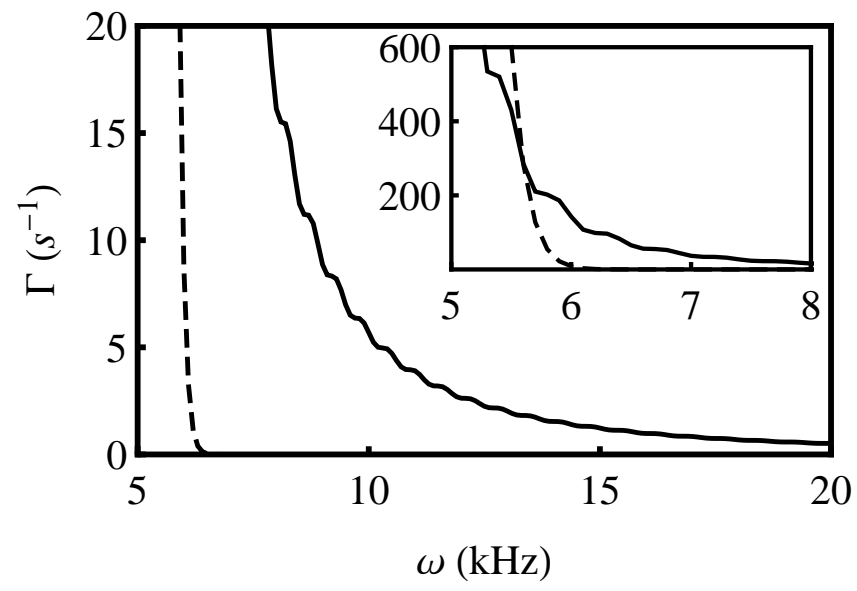

Figure 1: Heating rate as a function of the driving frequency for $\Omega_{R} / \omega=1$. The ground state corresponds to the Thomas Fermi approximation (continuous line), and, (in a single-particle description), to the ground state of a harmonic oscillator (dashed line). In the inset the vertical scale is enlarged.

\section{B. The decay of the excited states: characterization of the elementary excitations}

The nonstationary character of the excited state must be taken into account. In the description that includes atomic interaction effects, the final state in the interband transition will be shown to be embedded in the set of elementary excitations of the condensate, and, coupled to them. As a result, a damping mechanism, standardly known as Beliaev or Landau damping, turns up; it basically consists in nonlinear mode-coupling, i.e., in interaction between modes rooted in scattering terms of order higher than quadratic, (not included in the mode characterization). We proceed to trace this mechanism in our system.

We will depart from the diagonalization of a quadratic approximation to our manyparticle zero-order Hamiltonian, i.e., to the generalization of $H_{0}$ obtained by adding the inter-atomic potential [16, 17]. Subsequently, we will build a perturbative scheme, where corrections to the quadratic setup can be incorporated. Those additional terms account for coupling between the unperturbed states. The resulting mixing of modes will be introduced into an operative procedure to evaluate the heating rate through the modification, by a shift and a width, of the energy of the state characterized as $\left|\varphi^{(f)}\right\rangle$ in Eq. (12). The shift implies the redefinition of the resonance, and the width incorporates the decay. In this form, the description given by Eq. (12) is extended in the unperturbed-state representation. 
Alternatively, one could switch to the picture of eigenstates of the perturbed system and regard the mixing as leading to the enlargement of the range of the states that can be reached from the initial state through $H_{1}$. Then, damping should be incorporated into Eq. (12) via the modification of the coupling matrix-element and of the effective density of states [18].

Useful to isolate the basic components of damping is to tackle a simplified model. We will consider a uniform system. The effects of the harmonic confinement will be discussed later on. Additionally, the interaction potential will be simply characterized by the scattering lengths, as in usual ultra-cold-atom contexts. The driving-dependent scattering terms that emerge from the application of the unitary transformation given by Eq. (3) will be analyzed further on. By now, we deal only with the (dominant) static scattering potential. For the considered set of SOC parameters, $\left(\delta_{0}=0\right.$ and $\left.\Omega_{0}=0\right)$, the expression for the energy bands, obtained from Eq. (8), reads

$$
E_{ \pm}\left(k_{x}\right)=\frac{\hbar^{2}\left(k_{x} \mp k_{D}\right)^{2}}{2 m}+E_{L}+E_{D}
$$

where we have defined $k_{D}=\frac{\alpha m}{\hbar^{2}}$ and $E_{D}=-\frac{\hbar^{2} k_{D}^{2}}{2 m}$. We recall that $\alpha=J_{0}\left(\frac{\Omega_{R}}{\omega}\right) \alpha_{0}=$ $J_{0}\left(\frac{\Omega_{R}}{\omega}\right) \frac{2 E_{L}}{k_{L}}$. Then, the complete system can be regarded as composed by two uniform subsystems displaced in different directions in the quasi-momentum quantity $k_{D}$ (see Fig. 2).

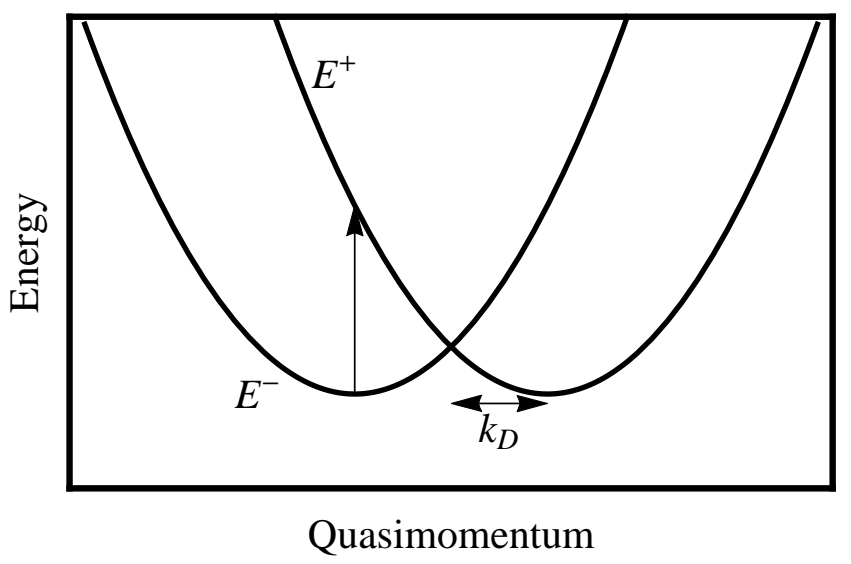

Figure 2: Diagram of energy bands versus quasi-momentum. $\left(\delta_{0}=0\right.$ and $\left.\Omega_{0}=0\right)$.

As corresponds to the assumed SOC parameters and to the values of $g_{\eta, \eta^{\prime}}$ for the referred realization, we consider a condensate in the (left) band $E_{-}\left(k_{x}\right)$. The term $H_{1}$ induces transitions to states in the other (right) band $E_{+}\left(k_{x}\right)$, which, initially, is not populated. Those transitions are illustrated in Fig. 2. The final states are denoted as $\left|\varphi_{+, \mathbf{k}}^{(f)}\right\rangle$, i.e., we 
specify the well-defined momentum (a uniform confinement is being considered), and the band label. Because of the preparation and the simplified band-structure, the elementary excitations can be described via a direct generalization of those corresponding to the nonSOC scenario. The Hamiltonian is written in terms of the operators $a_{\eta, \mathbf{k}}^{\dagger}\left(a_{\eta, \mathbf{k}}\right)$, which correspond to the creation (annihilation) of a particle in the state $|\eta, \mathbf{k}\rangle$, where $\eta$ stands for the band $(+,-)[($ right, left $)]$ and $\mathbf{k}$ denotes the quasi-momentum. The characterization of the eigenstates of the right subsystem is complete at this point: since a single-particle description is feasible, the operator $a_{+, \mathbf{k}}^{\dagger}$ simply corresponds to the creation of a particle in the state $\left|\varphi_{+, \mathbf{k}}\right\rangle$. In contrast, for the left-band subsystem, our procedure continues: the Bogolubov approach is applied to obtain the quasiparticles [16, 17]. This separate characterization is possible since there are no (interband) terms in the quadratic approximation. We will see that it is from the cubic corrections that interband coupling emerges. Displaced quasimomenta in the $x$-direction are used, i.e., we take in the left band $(-) \tilde{k}_{x}=k_{x}+k_{D}, \tilde{k}_{y}=k_{y}$, $\tilde{k}_{z}=k_{z}$, and write

$$
\begin{aligned}
b_{-, \tilde{\mathbf{k}}} & =u_{\tilde{k}} a_{-, \tilde{\mathbf{k}}}+v_{\tilde{k}} a_{-,-\tilde{\mathbf{k}}}^{\dagger} \\
b_{-,-\tilde{\mathbf{k}}}^{\dagger} & =u_{\tilde{k}} a_{-,-\tilde{\mathbf{k}}}^{\dagger}+v_{\tilde{k}} a_{-, \tilde{\mathbf{k}}}
\end{aligned}
$$

where $b_{-, \tilde{\mathbf{k}}}^{\dagger}\left(b_{-, \tilde{\mathbf{k}}}\right)$ denotes the creation (annihilation) operator of a quasi-particle, and $u_{\tilde{k}}$ and $v_{\tilde{k}}$ are real coefficients, which are standardly obtained. The associated (unperturbed) energies are given by

$$
E_{-, \tilde{k}}=\sqrt{\frac{(\hbar \tilde{k})^{2}}{2 m}\left[\frac{(\hbar \tilde{k})^{2}}{2 m}+2 \rho g\right]}
$$

where $\rho=N / \mathcal{V}$ is the particle density, ( $N$ denotes the number of particles in the condensate and $\mathcal{V}$ is the effective volume of confinement). Moreover, $g$ denotes the interaction strength of the atoms in the condensate. It is $g_{\downarrow \downarrow} \equiv g$ that appears in Eq. (16), as we are describing the left band, where the condensate has been prepared.

The above reduction of the system is feasible because of the simplifications applicable to the considered set of SOC parameters. For a generic regime, the characterization of the elementary excitations is a much more involved problem. 


\section{The effect of nonlinear mode-coupling}

The eigenstates of the quadratic Hamiltonian for the left-band subsystem, characterized by the operators $b_{-, \tilde{\mathbf{k}}}$, are actually coupled through the cubic and quartic terms, left out in the above (zero-order) approximation. They are also coupled to the eigenstates of the right-band subsystem, i.e., to the single-particle states $\left|\varphi_{+, \tilde{\mathbf{k}}}\right\rangle$. We have changed their labels by using the displaced momenta. The same notation will be applied to the corresponding

operators $a_{+, \tilde{\mathbf{k}}}^{\dagger}\left(a_{+, \tilde{\mathbf{k}}}\right)$. Since we are interested in the decay of the states $\left|\varphi_{+, \tilde{\mathbf{k}}}\right\rangle$, we will focus on the (leading) cubic terms that include them. In second quantization, those terms have the generic form

$$
V_{i n t}^{(3)} \sim \sqrt{N} g_{\uparrow \downarrow}\left(u_{\tilde{k}^{\prime}}-v_{\tilde{k}^{\prime}}\right) b_{-, \tilde{\mathbf{k}}^{\prime}}^{\dagger} a_{+, \tilde{\mathbf{k}}-\tilde{\mathbf{k}}^{\prime}}^{\dagger} a_{+, \tilde{\mathbf{k}}},
$$

which defines a two-particle scattering where one of the input particles is in $\left|\varphi_{+, \tilde{\mathbf{k}}}\right\rangle$ and the other in the condensate, the output states being $\left|\varphi_{+, \tilde{\mathbf{k}}-\tilde{\mathbf{k}}^{\prime}}\right\rangle$ and the quasi-particle created by $b_{-, \tilde{\mathbf{k}}^{\prime}}^{\dagger} \cdot\left(g_{\uparrow \downarrow}\right.$ is the interaction strength relevant to that process). Momentum conservation has been incorporated into the notation; energy conservation implies an additional restriction on the particle momenta. The factor $\sqrt{N}$ appears in Eq. (17) because one of the interacting particles is in the condensate. Since the quartic terms correspond to scattering with no particles in the condensate, the magnitude of their correction to the heating rate can be anticipated to be a factor $N$ smaller than that associated with $V_{\text {int }}^{(3)}$.

The extension of the quadratic setup by incorporating cubic terms leads to the decay of the unperturbed states [19-22]. Beliaev and Landau damping processes are variations of this physical mechanism [21, 23-25]. In Beliaev damping, an excited mode decays into two lower ones. In Landau damping, an upper state is reached via collisions between thermallypopulated lower modes. The relative importance of those processes depends on the temperature and the density of accessible output modes. In the scenario considered here, the highly-excited character of the decaying state implies the existence of a large number of accessible output modes, and, consequently, the potentially important role of Beliaev damping. Moreover, because of the very low temperature considered, the initial thermal populations of excited modes can be neglected, and, therefore, the secondary character of Landau damping can be conjectured.

Through a standard perturbative treatment, the effect of the terms $V_{i n t}^{(3)}$, (in a Beliaev- 
damping scenario), is incorporated into our description as a modification of the energy of the final state, which is is written as

$$
\mathcal{E}_{-}\left(k_{x}\right)=\frac{\hbar^{2} k_{x}^{2}}{2 m}+E_{L}+E_{D}+\delta_{k_{x}}-i \hbar \frac{\gamma_{k_{x}}}{2} .
$$

Here, the unperturbed value appears corrected by the effective width $\gamma_{k_{x}}$ and shift $\delta_{k_{x}}$, which can be evaluated analytically in a highly-diluted regime, i.e., for $\left(\rho a^{3}\right)^{1 / 2} \ll 1$, where $a$ corresponds to any of the involved scattering lengths.

The modification of Eq. (12) to incorporate the decay of the final state is straightforward [18]. In the case of a uniform model for the ground state, the heating rate is given by

$$
\Gamma(\omega)=\pi J_{1}^{2}\left(\frac{\Omega_{R}}{\omega}\right)\left(\alpha_{0} k_{D}\right)^{2} \frac{\gamma_{k_{x}}}{\left[\Delta E\left(k_{D}\right)-\hbar \omega\right]^{2}+\left(\hbar \frac{\gamma_{k_{x}}}{2}\right)^{2}} .
$$

$\left[\Delta E\left(k_{D}\right) \equiv E_{+}\left(-k_{D}\right)-E_{-}\left(-k_{D}\right)\right]$. The effect of the shift $\delta_{k_{x}}$ has been neglected: due to the high frequency of the driving, the shift in the energy hardly modifies the decay rate. The generalization of the above expression to a nonuniform model for the ground state is direct. Damping obtained using appropriate values for the density and scattering lengths in the system is incorporated into Figs. 3 to 8.

Our results for damping at high energies, where the elementary excitations have singleparticle character, can be reasonably expected to be robust when trapping effects are incorporated. In that range, the uniform approximation can be assumed to incorporate the essential components, the harmonic effects being expected to merely modify the form of the states, and, consequently, the coupling matrix elements. In contrast, the trapping conditions cannot be avoided in the analysis of the collective modes generated by the imperfect adiabatic character of the ramp. The distribution of frequencies is determined by the trap characteristics. The observed persistence of those low-frequency oscillations can be understood taking into account their small damping rate. Indeed, in the low energy-regime, where the elementary excitations have collective character, Beliaev damping is negligible: the number of accessible (output) modes is significantly reduced due to the restrictions that the discrete character of the spectrum adds to the conservation of energy and momentum. It is Landau damping, proportional to the temperature in the considered low-temperature regime, that can be assumed to account for the observed (weak) damping.

The presence of $\gamma_{k_{x}}$ in Eq. (19) indicates that the decay can significantly affect the width of the instability region. This is reflected in Fig. 3, where $\Gamma(\omega)$, obtained using 
different models, is represented. Actually, the appearance of the damping effects varies with the ground-state characteristics: it is the relative importance of damping and widening due to the momentum distribution that determines the form of the curves. Namely, the predictions, presented in Fig. 1, for the (nondissipative) Thomas-Fermi model are slightly modified by damping. In contrast, significant changes take place when variations of the ground-state model are considered: damping appreciably broadens the rate corresponding to characterizations of the ground state as a plane wave and as a harmonic-oscillator state. Moreover, in those cases, where the role of damping in widening the function $\Gamma(\omega)$ dominates over the effect of the momentum distribution, the precise modeling of the ground state becomes irrelevant, as can be seen in Fig. 4. Special attention requires the analysis of the results for $\Gamma\left(\Omega_{R} / \omega\right)$. The central importance of the reduced amplitude $\Omega_{R} / \omega$ in our description is reflected in the curves in Figs. 4 and 5. The separate presentation of the results for the plane wave and harmonic-oscillator ground state (Fig. 4) and those of the Thomas-Fermi distribution (Fig. 5) is imposed by the widely different magnitude of them. In passing, those results emphasize the need of including atomic-interaction effects to account for the experimental findings. We must clearly separate in those curves the features that are specific to the used set of parameters from those of general relevance. Particularly important is to trace the appearance of zeros in the heating rate. As formerly discussed, in a general regime, it is the factor $J_{1}^{2}\left(\Omega_{R} / \omega\right)$ that is certainly known to determine the magnitude of heating. Then, irrespective of the set of parameters, the oscillations of $J_{1}\left(\Omega_{R} / \omega\right)$ can be expected to be relevant to the form of $\Gamma\left(\Omega_{R} / \omega\right)$. In particular, the zeros of $J_{1}\left(\Omega_{R} / \omega\right)$ trivially lead to the inhibition of the population transfer in a first-order perturbative treatment. The global form of the curves of $\Gamma\left(\Omega_{R} / \omega\right)$ presented in Fig. 4 indicates the emergence of additional effects, rooted in the form of the coupling matrix-elements. The additional zeros that appear in $\Gamma\left(\Omega_{R} / \omega\right)$ are specific to the particular set of parameters considered: they correspond to zeros of $J_{0}\left(\Omega_{R} / \omega\right)$, as can be checked by an approximate analytic evaluation of the matrix elements. They cannot be used to curb the effects of heating simply because, for the corresponding driving parameters, there is no SOC in the zero-order Hamiltonian. 


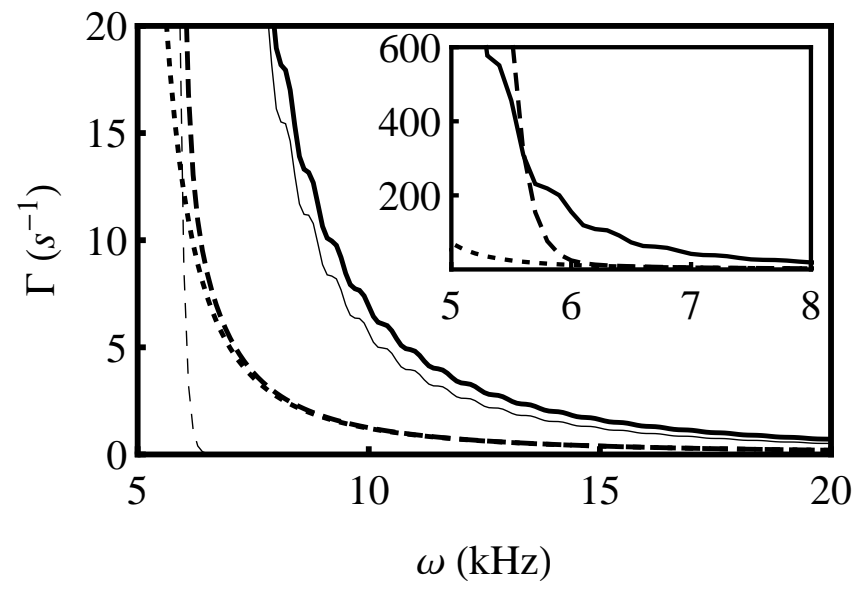

Figure 3: Heating rate as a function of the driving frequency for $\Omega_{R} / \omega=1$. The ground state corresponds to the Thomas Fermi approximation (continuous line), to the ground state of a harmonic oscillator (dashed line), and to a plane wave (dotted line). Thick lines incorporate damping, thin lines correspond to the nondissipative system (data from Fig. 1). In the inset the vertical axis is enlarged.

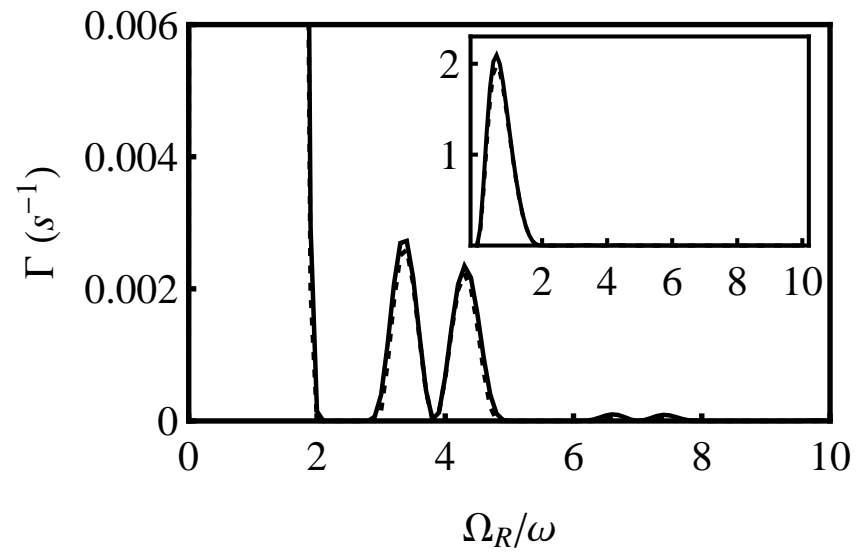

Figure 4: Heating rate as a function of the reduced driving amplitude $\Omega_{R} / \omega$ for $\omega=10 \mathrm{kHz}$. The ground state has been modeled as a plane wave (continuous line) and as the ground state of a harmonic oscillator (dashed line). Damping has been included. In the inset the vertical axis is enlarged.

We have assumed that our first-order approach provides a sufficiently sound framework to calculate the heating rates. This has been confirmed by evaluating the corrections introduced by higher-order perturbative terms, i.e., by $H_{n}$ with $n>1$. Fig. 5 provides a global view 
of the magnitude of those corrections. There, the validity of our framework is apparent. In our Figures, we have fixed the coupling strengths and the density to appropiate values. In fact, the dependence of the population loss and instability region on those parameters is evident: it can be simply traced back to the form of the cubic terms given by Eq. (17). In Fig. 6, results for $\Gamma$ as a function of $\Omega_{R} / \omega$, at three different frequencies, are presented. The decrease of the heating rate with increasing frequencies in the whole range for the reduced amplitude is evident. Hence, consistently with the experimental findings, the applicability of the scenario of control proposed in Ref. [7] is shown to be recovered in the high-frequency regime.

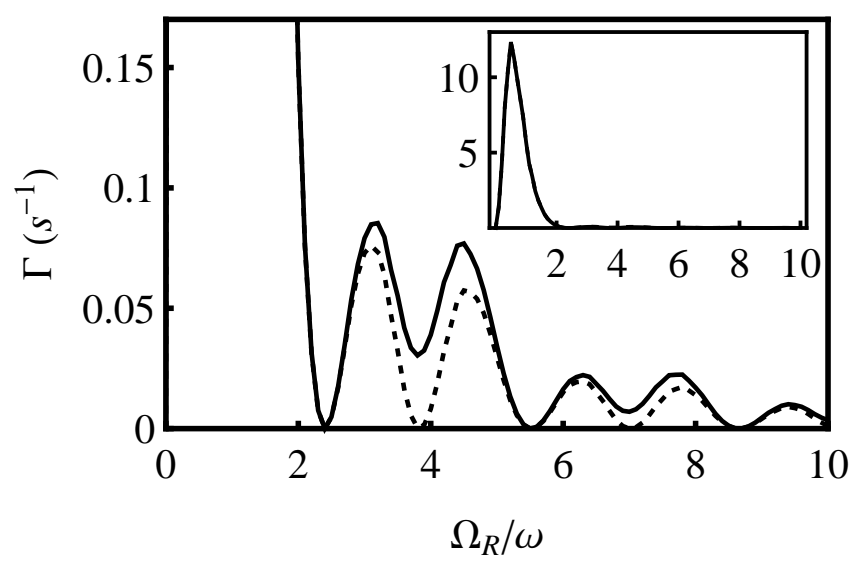

Figure 5: Heating rate as a function of the reduced driving amplitude $\Omega_{R} / \omega$ for $\omega=10 \mathrm{kHz}$, obtained including only the dominant $H_{1}$ correction in the perturbative scheme (dashed line; data from Fig. 4) and with all corrections $H_{n}$ (continuous line). The ground state corresponds to the Thomas-Fermi approximation. Damping has been included. In the inset the vertical axis is enlarged. 


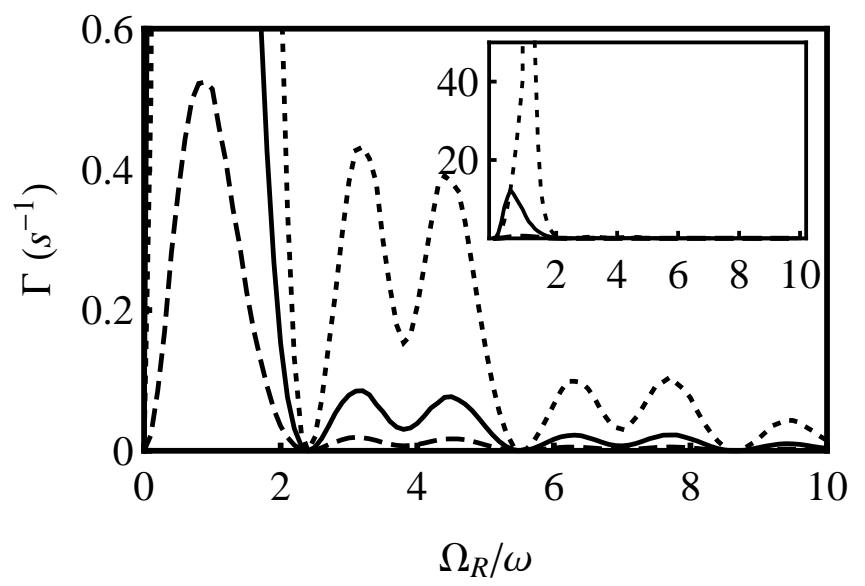

Figure 6: Heating rate as a function of the reduced driving amplitude $\Omega_{R} / \omega$ at three different frequencies: $\omega_{1}=5 \mathrm{kHz}$ (dotted line), $\omega_{2}=10 \mathrm{kHz}$ (continuous line), and $\omega_{3}=20 \mathrm{kHz}$ (dashed line). The ground state corresponds to the Thomas-Fermi approximation. Damping and all $H_{n}$ corrections have been included. In the inset the vertical axis is enlarged.

\section{Dressing of the scattering potential}

We turn to assess the relevance of having different interaction strengths, associated with the spin combinations. The spin-dependent scattering potential corresponding to the experimental setup, characterized by strengths which fulfill $g_{\uparrow \uparrow}=g_{\downarrow \uparrow} \neq g_{\downarrow \downarrow}$, is not invariant under the unitary transformation given by Eq. (3). In fact, the application of $U(t)$ to the interatomic potential leads to the emergence of driving-dependent terms which must be added to the static ones considered by now in the transformed many-body Hamiltonian. In particular, for the formerly considered SOC parameters and system preparation, the correction reads

$$
\frac{\Delta g}{4} \rho \sin [\zeta(t)](1-\cos [\zeta(t)]) \sigma_{y}
$$

where $\Delta g=g_{\uparrow \uparrow}-g_{\downarrow \downarrow}$. This term accounts for interband coupling, which can be relevant, again, in the quasiresonant case. Consequently, it can lead to heating. Note that the structure of the correction is similar to that of $H_{1}$, except in the absence of the dependence on $k_{x}$. Still, the induced transitions are vertical in momentum. We have evaluated the associated heating effects and found that, for the considered experimental conditions, they are orders of magnitude lower than those corresponding to $H_{1}$. We leave for future work the analysis of the potential control of this term via the appropriate choice of system and 
driving parameters and the use of Feshbach resonances. Note that the analyzed correction is an example of how the dressing of the atomic system by driving fields, (in the considered case, by the Raman-laser modulation), can modify the scattering characteristics. The same physics underlies the density-dependent synthetic gauge fields, recently found in a related context [26].

\section{HEATING IN THE RAMPING PROCESS}

We must incorporate into our description the modification of the parameters of $H_{0}$ that takes place in the ramp implemented to adiabatically vary $\Omega_{R}$ from zero to its final value. First, one must deal with the changing inter-band energy, and, in turn, with the varying character of the resonance between the driving frequency and the inter-band transition. Additionally, we must take into account that, the operator $W$, which depends on $\Omega_{R}$, varies during the ramping process. The potential changing magnitude of damping in different segments of the ramp must also be assessed. In the stages which correspond to a low-energy regime, damping is irrelevant: the Beliaev processes are blocked because of the reduced number of accessible output states. It is in the stages where significant inter-band splitting has been reached, that the (Beliaev) damping becomes important. As formerly stated, Landau damping is negligible due to the assumed low temperature. From the combination of those factors, the transition rate $\Gamma$ can present a nontrivial time-dependence, which can allow a variety of behaviors ranging from the absence of significant interband transfer in the whole ramp to strong heating in specific time intervals. This is shown in Fig. 7, where we depict the heating rate as a function of both, the frequency $\omega$, and the reduced amplitude $\Omega_{R} / \omega$. Cases a) and b) respectively correspond to modeling the ground state in the Thomas-Fermi approximation and as a harmonic-oscillator state. A ramp corresponds to a fixed $\omega$ and to an increasing $\Omega_{R} / \omega$. A first noticeable feature is, again, the robustness of the control strategy at high frequencies: losses are negligible along the whole ramp. As the frequency diminishes, heating becomes relevant, basically in an initial region for the (increasing) parameter $\Omega_{R} / \omega$. After that region, the population of the system in the lowest band remains stable. We recall that the zeros in $\Gamma\left(\Omega_{R} / \omega\right)$ rooted in those of $J_{1}\left(\Omega_{R} / \omega\right)$ are specifically associated with the inhibition of the population transfer. In contrast, the additional zeros that appear in $\Gamma\left(\Omega_{R} / \omega\right)$ can be traced to the considered particular set of SOC parameters; as previously 
indicated, since they derive from zeros of $J_{0}\left(\Omega_{R} / \omega\right)$, they correspond to a suppression of the SOC. Then, they cannot be used in a strategy for curbing heating in a general regime. An additional feature of the curves must be emphasized. Namely, at very low frequencies, the whole perturbative scheme breaks down: the zero-order Hamiltonian does not provide an appropriate framework for analyzing the dynamics.

To uncover the actual magnitude of heating we must integrate the losses along the complete ramp. In Fig. 8, we depict the corresponding energy gained in the band transfer as a function of the driving frequency, i.e., for different complete ramps. The ground state has been alternatively modeled using the Thomas-Fermi approach and as the one-particle Gaussian ground state. In the presentation of the experimental results, the heating was parametrized in terms of an effective temperature. Here, we do not tackle the thermalization of the system. In fact, this is a nontrivial problem in this context. Hence, we have opted for evaluating the heating directly from the gained energy. Our curves are shown to peak at the resonance-frequency region. Differences in the estimated width of the peak are rooted in the different distribution of momentum corresponding to the applied models. The results reveal that the system preparation can play a crucial role in heating: for ramps corresponding to a broad range of frequencies, significant losses of population take place before reaching the final value of $\Omega_{R}$. In the experiments, the heating was observed to reach a plateau at large frequencies. That feature is not reproduced in our approach, which focuses here on heating by interband transitions. We conjecture that feature to be rooted in additional heating mechanisms. The need of pondering these effects in the design of the strategy of control is apparent. An interesting subject of future work can be the design of alternative and more effective forms of ramping, where the implications of our analysis can be incorporated. Here, one can anticipate that a compromise must be reached between minimizing the relevance of the region of significant losses, for instance, by ramping faster there, and, on the other hand, curbing the corrections associated to the consequent departures from adiabaticity. The optimization of the ramp is out of the scope of the present paper. 
(a)

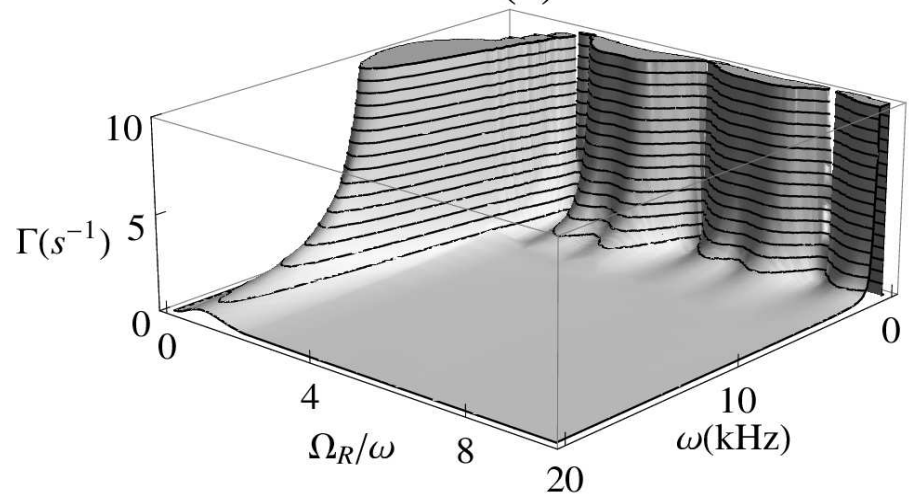

(b)

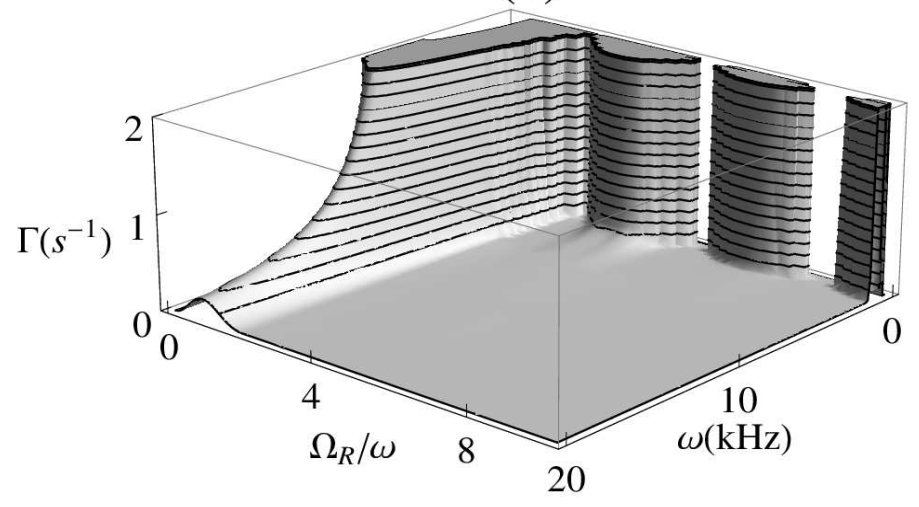

Figure 7: Heating rate as a function of the driving frequency $\omega$ and effective amplitude $\Omega_{R} / \omega$. The ground state is modeled with the Thomas-Fermi approximation (a) and as the ground state of a harmonic oscillator (b). Damping has been included. 


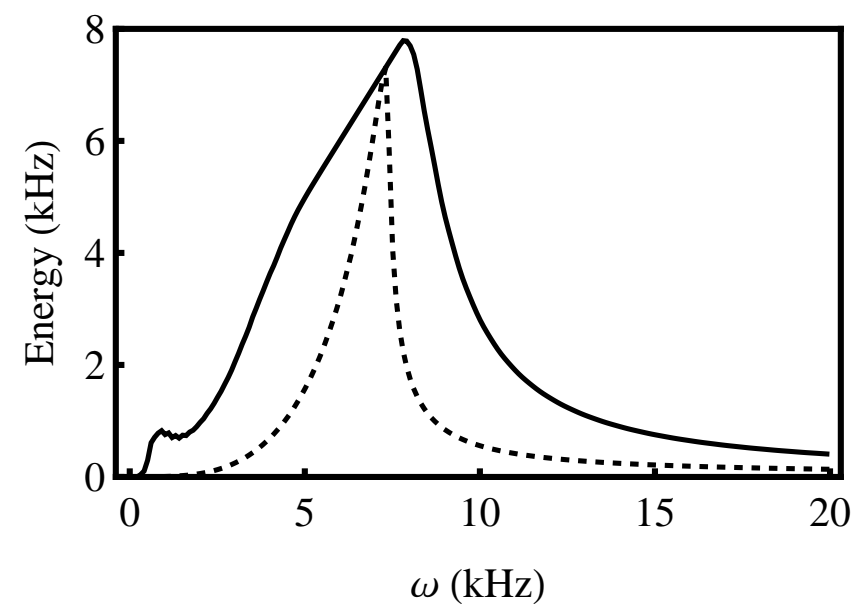

Figure 8: Energy gained in the heating process as a function of the driving frequency at $\Omega_{R} / \omega=2.9$. The ground state corresponds to the Thomas Fermi approximation (continuous line) and to the ground state of a harmonic oscillator (dashed line). Damping has been included.

\section{CONCLUDING REMARKS}

The study corroborates that the restrictions of working with high driving-frequencies and very slow variations in the modulation amplitude are sufficient for guaranteeing the applicability of the technique of control. In the high-frequency regime, the description of the system given by $H_{0}$ has been found to be valid, the effect of the higher-order corrections being then negligible. Moreover, it has been shown that the safe range of applicability can be extended. Namely, if practical limitations impose the use of smaller frequencies, a first general recommendation is to avoid the region of resonance between the modulation and the inter-band transition. This applies to any interval in the ramping processes. Still, in the resonance region, the adjustment of the quotient of parameters $\Omega_{R} / \omega$ to reduce the magnitude of the Bessel function $J_{1}\left(\Omega_{R} / \omega\right)$ can be used to curb heating. It is worth stressing, that the relevance of higher-order corrections to the Hamiltonian of control can be enhanced by the characteristics of the practical arrangement. For instance, in setups where a continuous variations of the band splitting is implemented, like in realizations of Landau-Zener analogs [27], different higher-order terms can become dominant if the sequence of resonance windows is crossed. Given the broad use of similar averaging techniques to obtain reduced pictures in different contexts, our scheme for suppressing the effect of the 
perturbations at different orders can be expected to have quite general applicability. In the system considered, there are no technical problems in the realization of this strategy. The analysis has also uncovered the potential relevance of the ramp to heating: depending on the driving frequency, significant losses of population can occur before reaching the intended value of the driving amplitude. In addition to curbing the inter-band transfer of population, the methods of control must prevent the generation of intra-band excitations. In this regard, departures from adiabaticity in the variation of the modulation amplitude must be minimized. The development of efficient alternative forms of the ramp which can prevent the crossing of the resonance region and the excitation of collective modes constitutes an interesting line for future research.

The possibility of using the driving, not only as an element of control, but as an instrument to explore the excited modes is open. In parallel, the development of models applicable to the trapped system can benefit from the conclusions of our study. Future work will be dedicated to the characterization of the elementary excitations in regimes for the SOC parameters where the simplifications assumed in our work are not applicable. Significant advances in this line can result from the availability of a controllable testing ground for the theoretical predictions.

In the studied system, there is little difference between the scattering strengths associated with the different spin combinations. As a consequence, the analysis of the role of the atomicinteraction potential simplifies considerably. However, one can think of realizations where a larger variety of scattering lengths can be used to increase the versatility of the scenario. In fact, our procedure has uncovered that the spin dependence of both, the interaction potential and the driving term in the Hamiltonian, leads to density dependent contributions to heating. Hence, nontrivial effects can be expected from significant differences between the involved scattering lengths. Apart from being applicable to the Raman-laser scenario, the study presents arguments that can be relevant to other SOC setups [3], where dressing of the atomic systems by classical fields is used. Moreover, since significant characteristics of the heating have been traced to the single-particle dynamics, one can think of extrapolating part of the conclusions, those referring to the initial single-particle stages of our study, to parallel arrangements in fermionic systems. 


\section{Acknowledgments}

One of us (JMGL) acknowledges the support of the Spanish Ministerio de Economía y Competitividad and the European Regional Development Fund (Grant No. FIS 2013-41532$\mathrm{P})$.

[1] Y.-J. Lin, K. Jiménez-García, and I. B. Spielman, Nature (London) 471, 83 (2011).

[2] N. Goldman, G. Juzeliunas, P. Ohberg, and I. B. Spielman, Rep. Prog. Phys. 77, 126401 (2014).

[3] J. Dalibard, F. Gerbier, G. Juzeliunas, and P. Ohberg, Rev. Mod. Phys. 83, 1523 (2011).

[4] P. Wang, Z-Q. Yu, Z. Fu, J. Miao, L. Huang, S. Chai, H. Zhai, and J. Zhang, Phys. Rev. Lett. 109, $095301(2012)$.

[5] L. W. Cheuk, A. T. Sommer, Z. Hadzibabic, T. Yefsah, W. S. Bakr, and M. W. Zwierlein, Phys. Rev. Lett. 109, 095302 (2012).

[6] Y. Zhang, G. Chen, and C. Zhang, Sci. Rep. 3, 1937 (2013).

[7] K. Jiménez-García et al., Phys. Rev. Lett. 114, 125301 (2015). (See also the Supplemental Material.)

[8] Y. Li, G.I. Martone, and S. Stringari (2015) Spin-Orbit-coupled Bose-Einstein condensates. Annual Review of Cold Atoms and Molecules: pp. 201-250.

[9] Y. Li, L. P. Pitaevskii, and S. Stringari, Phys. Rev. Lett. 108, 225301 (2012).

[10] Y. Li, G. I. Martone, L. P. Pitaevskii, and S. Stringari, Phys. Rev. Lett. 110, 235302 (2013).

[11] I. B. Spielman, Phys. Rev. A 79, 063613 (2009).

[12] W.H. Louisell, Quantum Statistical Properties of Radiation (John Wiley, New York, 1973).

[13] I. S. Gradshteyn and I. M. Ryshik, Table of Integrals, Series, and Products (Academic Press, New York, 1994).

[14] C. Cohen-Tannoudji, B. Diu, and F. Laloe, Quantum Mechanics, (John Wiley, New York, 1977).

[15] Tin-Lun Ho and Shizhong Zhang, Phys. Rev. Lett. 107, 150403 (2011).

[16] L. P. Pitaevskii and S. Stringari, Bose-Einstein Condensation. (Oxford University Press, Oxford, 2003). 
[17] C.J. Pethick and H. Smith, Bose-Einstein Condensation in Dilute Gases, (Cambridge University Press, Cambridge, 2002).

[18] C. Cohen-Tannoudji, J. Dupont-Roc, and G. Grynberg, Atom-Photon Interactions (John Wiley \& Sons, Inc., New York, 1992).

[19] S. T. Beliaev, Sov. Phys.-JETP 7, 289 (1958).

[20] S. T. Beliaev, Sov. Phys.-JETP 7, 299 (1958).

[21] Shai Ronen, J. Phys. B: At. Mol. Opt. Phys. 42, 055301 (2009).

[22] F. Mohling and A. Sirlin, Phys. Rev. 118, 370 (1960).

[23] L. P. Pitaevskii and S. Stringari, Phys. Lett. A 235, 398 (1997).

[24] P. O. Fedichev, G. V. Shlyapnikov, and J. T. M. Walraven, Phys. Rev. Lett. 80, 2269 (1998).

[25] S. Giorgini, Phys. Rev. A 57, 2949 (1998).

[26] M. J. Edmonds, M. Valiente, G. Juzeliunas, L. Santos, and P. Ohberg, Phys. Rev. Lett. 110, 085301 (2013).

[27] A. J. Olson, Su-Ju Wang, R. J. Niffenegger, Chuan-Hsun Li, C. H. Greene, and Y. P. Chen, Phys. Rev. A 90, 013616 (2014). 